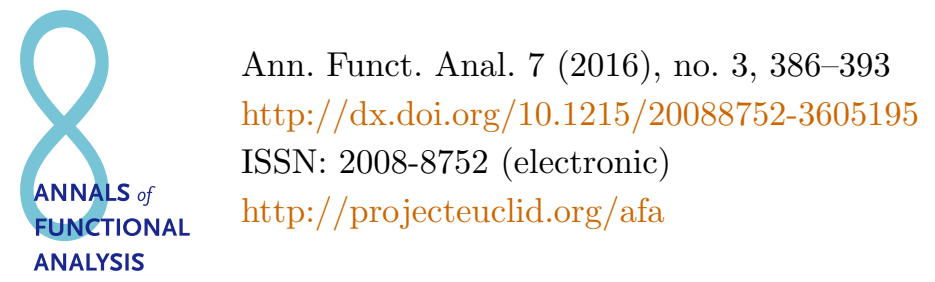

\title{
POLYTOPES OF STOCHASTIC TENSORS
}

\author{
HAIXIA CHANG,${ }^{1}$ VEHBI E. PAKSOY,${ }^{2}$ and FUZHEN ZHANG ${ }^{2 *}$ \\ Communicated by Q.-W. Wang
}

\begin{abstract}
Considering $n \times n \times n$ stochastic tensors $\left(a_{i j k}\right)$ (i.e., nonnegative hypermatrices in which every sum over one index $i, j$, or $k$, is 1$)$, we study the polytope $\left(\Omega_{n}\right)$ of all these tensors, the convex set $\left(L_{n}\right)$ of all tensors in $\Omega_{n}$ with some positive diagonals, and the polytope $\left(\Delta_{n}\right)$ generated by the permutation tensors. We show that $L_{n}$ is almost the same as $\Omega_{n}$ except for some boundary points. We also present an upper bound for the number of vertices of $\Omega_{n}$.
\end{abstract}

\section{IntRoduction}

A square matrix is doubly stochastic if its entries are all nonnegative and each row and column sum is 1. A celebrated result known as Birkhoff's theorem about doubly stochastic matrices (see, e.g., [8, p. 549]) states that an $n \times n$ matrix is doubly stochastic if and only if it is a convex combination of some $n \times n$ permutation matrices. Considered as elements in $\mathbb{R}^{n^{2}}$, the $n \times n$ doubly stochastic matrices form a polytope $\left(\omega_{n}\right)$. The Birkhoff's theorem says that the polytope $\omega_{n}$ is the same as the polytope $\left(\delta_{n}\right)$ generated by the permutation matrices. A traditional proof of this result is to make use of a lemma which ensures that every doubly stochastic matrix has a positive diagonal (see, e.g., [8, Lemma 8.7.1, p. 548]). By a "diagonal of an $n$-square matrix" we mean a set of $n$ entries taken from different rows and columns. The $n$-square doubly stochastic matrices having a positive diagonal form a polytope $\left(l_{n}\right)$ too. Apparently, $\delta_{n} \subseteq l_{n} \subseteq \omega_{n}$. Birkhoff's theorem asserts that the three polytopes $\omega_{n}, l_{n}$, and $\delta_{n}$ coincide.

Copyright 2016 by the Tusi Mathematical Research Group.

Received Sep. 18, 2015; Accepted Nov. 26, 2015.

${ }^{*}$ Corresponding author.

2010 Mathematics Subject Classification. Primary 15B51; Secondary 52B11.

Keywords. doubly stochastic matrix, extreme point, polytope, stochastic semi-magic cube, stochastic tensor. 


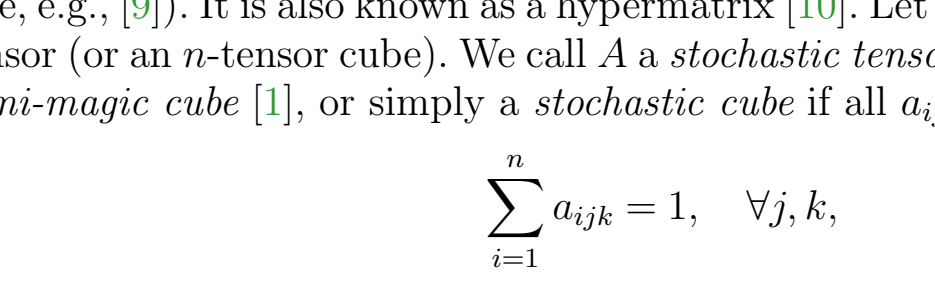

\title{
POLYTOPES OF STOCHASTIC TENSORS
}

\author{
HAIXIA CHANG,${ }^{1}$ VEHBI E. PAKSOY,${ }^{2}$ and FUZHEN ZHANG ${ }^{2 *}$ \\ Communicated by Q.-W. Wang
}

\begin{abstract}
Considering $n \times n \times n$ stochastic tensors $\left(a_{i j k}\right)$ (i.e., nonnegative hypermatrices in which every sum over one index $i, j$, or $k$, is 1$)$, we study the polytope $\left(\Omega_{n}\right)$ of all these tensors, the convex set $\left(L_{n}\right)$ of all tensors in $\Omega_{n}$ with some positive diagonals, and the polytope $\left(\Delta_{n}\right)$ generated by the permutation tensors. We show that $L_{n}$ is almost the same as $\Omega_{n}$ except for some boundary points. We also present an upper bound for the number of vertices of $\Omega_{n}$.
\end{abstract}

\section{IntRoduction}

A square matrix is doubly stochastic if its entries are all nonnegative and each row and column sum is 1. A celebrated result known as Birkhoff's theorem about doubly stochastic matrices (see, e.g., [8, p. 549]) states that an $n \times n$ matrix is doubly stochastic if and only if it is a convex combination of some $n \times n$ permutation matrices. Considered as elements in $\mathbb{R}^{n^{2}}$, the $n \times n$ doubly stochastic matrices form a polytope $\left(\omega_{n}\right)$. The Birkhoff's theorem says that the polytope $\omega_{n}$ is the same as the polytope $\left(\delta_{n}\right)$ generated by the permutation matrices. A traditional proof of this result is to make use of a lemma which ensures that every doubly stochastic matrix has a positive diagonal (see, e.g., [8, Lemma 8.7.1, p. 548]). By a "diagonal of an $n$-square matrix" we mean a set of $n$ entries taken from different rows and columns. The $n$-square doubly stochastic matrices having a positive diagonal form a polytope $\left(l_{n}\right)$ too. Apparently, $\delta_{n} \subseteq l_{n} \subseteq \omega_{n}$. Birkhoff's theorem asserts that the three polytopes $\omega_{n}, l_{n}$, and $\delta_{n}$ coincide.

Copyright 2016 by the Tusi Mathematical Research Group.

Received Sep. 18, 2015; Accepted Nov. 26, 2015.

${ }^{*}$ Corresponding author.

2010 Mathematics Subject Classification. Primary 15B51; Secondary 52B11.

Keywords. doubly stochastic matrix, extreme point, polytope, stochastic semi-magic cube, stochastic tensor. 


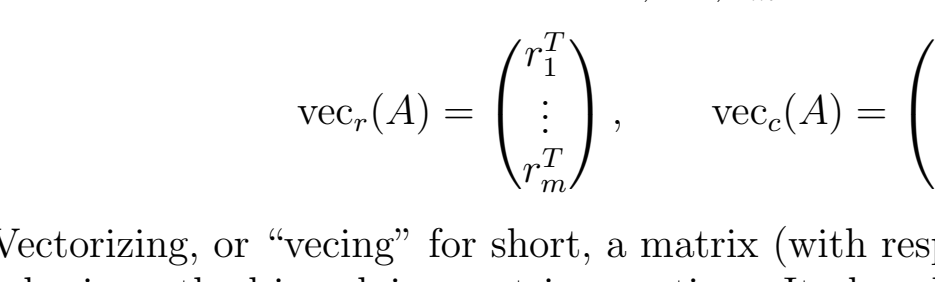

\title{
POLYTOPES OF STOCHASTIC TENSORS
}

\author{
HAIXIA CHANG,${ }^{1}$ VEHBI E. PAKSOY,${ }^{2}$ and FUZHEN ZHANG ${ }^{2 *}$ \\ Communicated by Q.-W. Wang
}

\begin{abstract}
Considering $n \times n \times n$ stochastic tensors $\left(a_{i j k}\right)$ (i.e., nonnegative hypermatrices in which every sum over one index $i, j$, or $k$, is 1$)$, we study the polytope $\left(\Omega_{n}\right)$ of all these tensors, the convex set $\left(L_{n}\right)$ of all tensors in $\Omega_{n}$ with some positive diagonals, and the polytope $\left(\Delta_{n}\right)$ generated by the permutation tensors. We show that $L_{n}$ is almost the same as $\Omega_{n}$ except for some boundary points. We also present an upper bound for the number of vertices of $\Omega_{n}$.
\end{abstract}

\section{IntRoduction}

A square matrix is doubly stochastic if its entries are all nonnegative and each row and column sum is 1. A celebrated result known as Birkhoff's theorem about doubly stochastic matrices (see, e.g., [8, p. 549]) states that an $n \times n$ matrix is doubly stochastic if and only if it is a convex combination of some $n \times n$ permutation matrices. Considered as elements in $\mathbb{R}^{n^{2}}$, the $n \times n$ doubly stochastic matrices form a polytope $\left(\omega_{n}\right)$. The Birkhoff's theorem says that the polytope $\omega_{n}$ is the same as the polytope $\left(\delta_{n}\right)$ generated by the permutation matrices. A traditional proof of this result is to make use of a lemma which ensures that every doubly stochastic matrix has a positive diagonal (see, e.g., [8, Lemma 8.7.1, p. 548]). By a "diagonal of an $n$-square matrix" we mean a set of $n$ entries taken from different rows and columns. The $n$-square doubly stochastic matrices having a positive diagonal form a polytope $\left(l_{n}\right)$ too. Apparently, $\delta_{n} \subseteq l_{n} \subseteq \omega_{n}$. Birkhoff's theorem asserts that the three polytopes $\omega_{n}, l_{n}$, and $\delta_{n}$ coincide.

Copyright 2016 by the Tusi Mathematical Research Group.

Received Sep. 18, 2015; Accepted Nov. 26, 2015.

${ }^{*}$ Corresponding author.

2010 Mathematics Subject Classification. Primary 15B51; Secondary 52B11.

Keywords. doubly stochastic matrix, extreme point, polytope, stochastic semi-magic cube, stochastic tensor. 


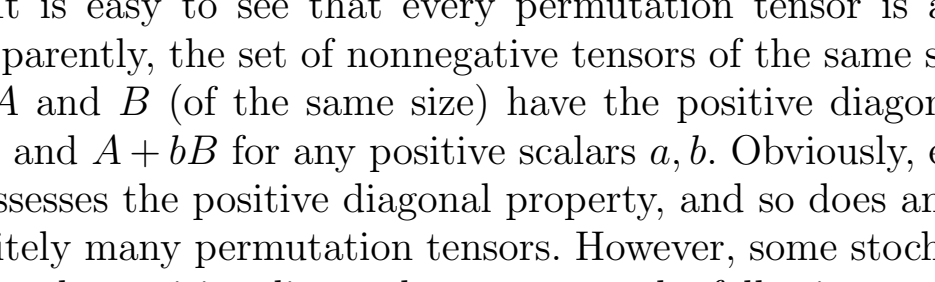

\title{
POLYTOPES OF STOCHASTIC TENSORS
}

\author{
HAIXIA CHANG,${ }^{1}$ VEHBI E. PAKSOY,${ }^{2}$ and FUZHEN ZHANG ${ }^{2 *}$ \\ Communicated by Q.-W. Wang
}

\begin{abstract}
Considering $n \times n \times n$ stochastic tensors $\left(a_{i j k}\right)$ (i.e., nonnegative hypermatrices in which every sum over one index $i, j$, or $k$, is 1$)$, we study the polytope $\left(\Omega_{n}\right)$ of all these tensors, the convex set $\left(L_{n}\right)$ of all tensors in $\Omega_{n}$ with some positive diagonals, and the polytope $\left(\Delta_{n}\right)$ generated by the permutation tensors. We show that $L_{n}$ is almost the same as $\Omega_{n}$ except for some boundary points. We also present an upper bound for the number of vertices of $\Omega_{n}$.
\end{abstract}

\section{IntRoduction}

A square matrix is doubly stochastic if its entries are all nonnegative and each row and column sum is 1. A celebrated result known as Birkhoff's theorem about doubly stochastic matrices (see, e.g., [8, p. 549]) states that an $n \times n$ matrix is doubly stochastic if and only if it is a convex combination of some $n \times n$ permutation matrices. Considered as elements in $\mathbb{R}^{n^{2}}$, the $n \times n$ doubly stochastic matrices form a polytope $\left(\omega_{n}\right)$. The Birkhoff's theorem says that the polytope $\omega_{n}$ is the same as the polytope $\left(\delta_{n}\right)$ generated by the permutation matrices. A traditional proof of this result is to make use of a lemma which ensures that every doubly stochastic matrix has a positive diagonal (see, e.g., [8, Lemma 8.7.1, p. 548]). By a "diagonal of an $n$-square matrix" we mean a set of $n$ entries taken from different rows and columns. The $n$-square doubly stochastic matrices having a positive diagonal form a polytope $\left(l_{n}\right)$ too. Apparently, $\delta_{n} \subseteq l_{n} \subseteq \omega_{n}$. Birkhoff's theorem asserts that the three polytopes $\omega_{n}, l_{n}$, and $\delta_{n}$ coincide.

Copyright 2016 by the Tusi Mathematical Research Group.

Received Sep. 18, 2015; Accepted Nov. 26, 2015.

${ }^{*}$ Corresponding author.

2010 Mathematics Subject Classification. Primary 15B51; Secondary 52B11.

Keywords. doubly stochastic matrix, extreme point, polytope, stochastic semi-magic cube, stochastic tensor. 


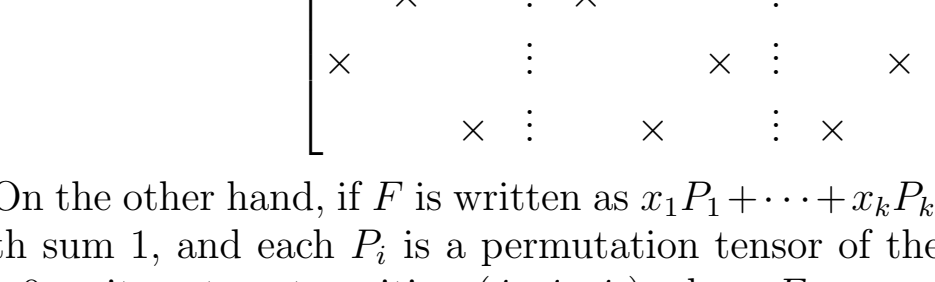

\title{
POLYTOPES OF STOCHASTIC TENSORS
}

\author{
HAIXIA CHANG,${ }^{1}$ VEHBI E. PAKSOY,${ }^{2}$ and FUZHEN ZHANG ${ }^{2 *}$ \\ Communicated by Q.-W. Wang
}

\begin{abstract}
Considering $n \times n \times n$ stochastic tensors $\left(a_{i j k}\right)$ (i.e., nonnegative hypermatrices in which every sum over one index $i, j$, or $k$, is 1$)$, we study the polytope $\left(\Omega_{n}\right)$ of all these tensors, the convex set $\left(L_{n}\right)$ of all tensors in $\Omega_{n}$ with some positive diagonals, and the polytope $\left(\Delta_{n}\right)$ generated by the permutation tensors. We show that $L_{n}$ is almost the same as $\Omega_{n}$ except for some boundary points. We also present an upper bound for the number of vertices of $\Omega_{n}$.
\end{abstract}

\section{IntRoduction}

A square matrix is doubly stochastic if its entries are all nonnegative and each row and column sum is 1. A celebrated result known as Birkhoff's theorem about doubly stochastic matrices (see, e.g., [8, p. 549]) states that an $n \times n$ matrix is doubly stochastic if and only if it is a convex combination of some $n \times n$ permutation matrices. Considered as elements in $\mathbb{R}^{n^{2}}$, the $n \times n$ doubly stochastic matrices form a polytope $\left(\omega_{n}\right)$. The Birkhoff's theorem says that the polytope $\omega_{n}$ is the same as the polytope $\left(\delta_{n}\right)$ generated by the permutation matrices. A traditional proof of this result is to make use of a lemma which ensures that every doubly stochastic matrix has a positive diagonal (see, e.g., [8, Lemma 8.7.1, p. 548]). By a "diagonal of an $n$-square matrix" we mean a set of $n$ entries taken from different rows and columns. The $n$-square doubly stochastic matrices having a positive diagonal form a polytope $\left(l_{n}\right)$ too. Apparently, $\delta_{n} \subseteq l_{n} \subseteq \omega_{n}$. Birkhoff's theorem asserts that the three polytopes $\omega_{n}, l_{n}$, and $\delta_{n}$ coincide.

Copyright 2016 by the Tusi Mathematical Research Group.

Received Sep. 18, 2015; Accepted Nov. 26, 2015.

${ }^{*}$ Corresponding author.

2010 Mathematics Subject Classification. Primary 15B51; Secondary 52B11.

Keywords. doubly stochastic matrix, extreme point, polytope, stochastic semi-magic cube, stochastic tensor. 


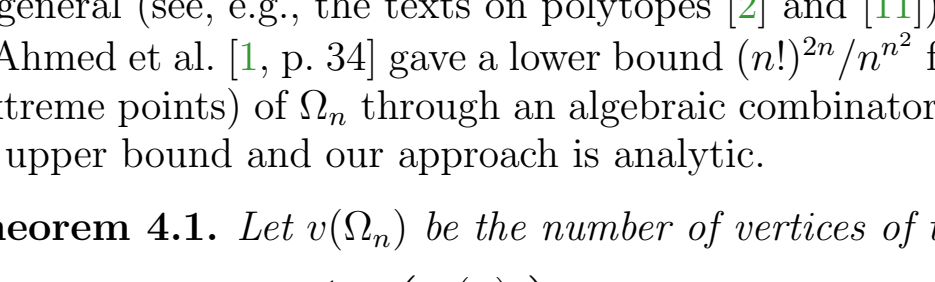

\title{
POLYTOPES OF STOCHASTIC TENSORS
}

\author{
HAIXIA CHANG,${ }^{1}$ VEHBI E. PAKSOY,${ }^{2}$ and FUZHEN ZHANG ${ }^{2 *}$ \\ Communicated by Q.-W. Wang
}

\begin{abstract}
Considering $n \times n \times n$ stochastic tensors $\left(a_{i j k}\right)$ (i.e., nonnegative hypermatrices in which every sum over one index $i, j$, or $k$, is 1$)$, we study the polytope $\left(\Omega_{n}\right)$ of all these tensors, the convex set $\left(L_{n}\right)$ of all tensors in $\Omega_{n}$ with some positive diagonals, and the polytope $\left(\Delta_{n}\right)$ generated by the permutation tensors. We show that $L_{n}$ is almost the same as $\Omega_{n}$ except for some boundary points. We also present an upper bound for the number of vertices of $\Omega_{n}$.
\end{abstract}

\section{IntRoduction}

A square matrix is doubly stochastic if its entries are all nonnegative and each row and column sum is 1. A celebrated result known as Birkhoff's theorem about doubly stochastic matrices (see, e.g., [8, p. 549]) states that an $n \times n$ matrix is doubly stochastic if and only if it is a convex combination of some $n \times n$ permutation matrices. Considered as elements in $\mathbb{R}^{n^{2}}$, the $n \times n$ doubly stochastic matrices form a polytope $\left(\omega_{n}\right)$. The Birkhoff's theorem says that the polytope $\omega_{n}$ is the same as the polytope $\left(\delta_{n}\right)$ generated by the permutation matrices. A traditional proof of this result is to make use of a lemma which ensures that every doubly stochastic matrix has a positive diagonal (see, e.g., [8, Lemma 8.7.1, p. 548]). By a "diagonal of an $n$-square matrix" we mean a set of $n$ entries taken from different rows and columns. The $n$-square doubly stochastic matrices having a positive diagonal form a polytope $\left(l_{n}\right)$ too. Apparently, $\delta_{n} \subseteq l_{n} \subseteq \omega_{n}$. Birkhoff's theorem asserts that the three polytopes $\omega_{n}, l_{n}$, and $\delta_{n}$ coincide.

Copyright 2016 by the Tusi Mathematical Research Group.

Received Sep. 18, 2015; Accepted Nov. 26, 2015.

${ }^{*}$ Corresponding author.

2010 Mathematics Subject Classification. Primary 15B51; Secondary 52B11.

Keywords. doubly stochastic matrix, extreme point, polytope, stochastic semi-magic cube, stochastic tensor. 


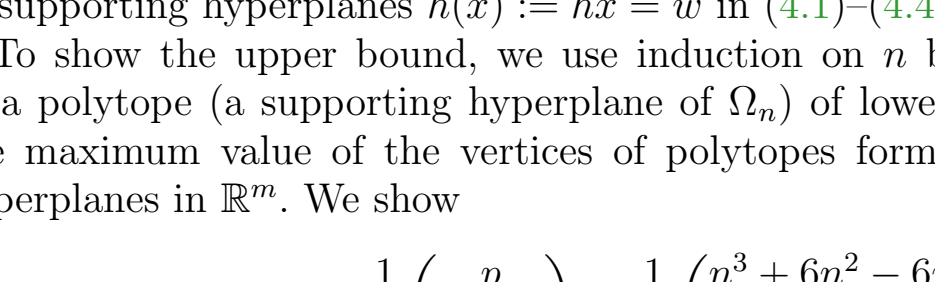

\title{
POLYTOPES OF STOCHASTIC TENSORS
}

\author{
HAIXIA CHANG,${ }^{1}$ VEHBI E. PAKSOY,${ }^{2}$ and FUZHEN ZHANG ${ }^{2 *}$ \\ Communicated by Q.-W. Wang
}

\begin{abstract}
Considering $n \times n \times n$ stochastic tensors $\left(a_{i j k}\right)$ (i.e., nonnegative hypermatrices in which every sum over one index $i, j$, or $k$, is 1$)$, we study the polytope $\left(\Omega_{n}\right)$ of all these tensors, the convex set $\left(L_{n}\right)$ of all tensors in $\Omega_{n}$ with some positive diagonals, and the polytope $\left(\Delta_{n}\right)$ generated by the permutation tensors. We show that $L_{n}$ is almost the same as $\Omega_{n}$ except for some boundary points. We also present an upper bound for the number of vertices of $\Omega_{n}$.
\end{abstract}

\section{IntRoduction}

A square matrix is doubly stochastic if its entries are all nonnegative and each row and column sum is 1. A celebrated result known as Birkhoff's theorem about doubly stochastic matrices (see, e.g., [8, p. 549]) states that an $n \times n$ matrix is doubly stochastic if and only if it is a convex combination of some $n \times n$ permutation matrices. Considered as elements in $\mathbb{R}^{n^{2}}$, the $n \times n$ doubly stochastic matrices form a polytope $\left(\omega_{n}\right)$. The Birkhoff's theorem says that the polytope $\omega_{n}$ is the same as the polytope $\left(\delta_{n}\right)$ generated by the permutation matrices. A traditional proof of this result is to make use of a lemma which ensures that every doubly stochastic matrix has a positive diagonal (see, e.g., [8, Lemma 8.7.1, p. 548]). By a "diagonal of an $n$-square matrix" we mean a set of $n$ entries taken from different rows and columns. The $n$-square doubly stochastic matrices having a positive diagonal form a polytope $\left(l_{n}\right)$ too. Apparently, $\delta_{n} \subseteq l_{n} \subseteq \omega_{n}$. Birkhoff's theorem asserts that the three polytopes $\omega_{n}, l_{n}$, and $\delta_{n}$ coincide.

Copyright 2016 by the Tusi Mathematical Research Group.

Received Sep. 18, 2015; Accepted Nov. 26, 2015.

${ }^{*}$ Corresponding author.

2010 Mathematics Subject Classification. Primary 15B51; Secondary 52B11.

Keywords. doubly stochastic matrix, extreme point, polytope, stochastic semi-magic cube, stochastic tensor. 


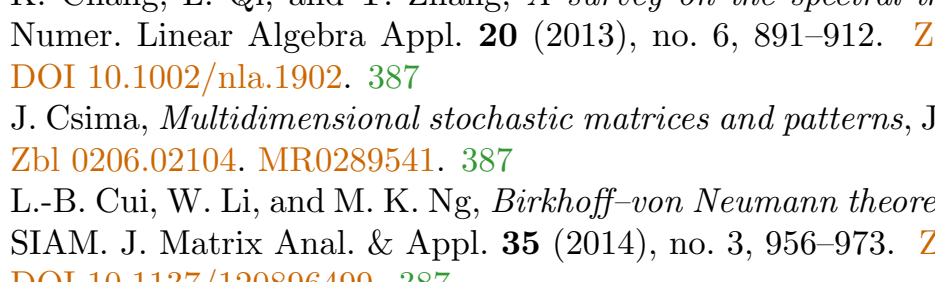

\title{
POLYTOPES OF STOCHASTIC TENSORS
}

\author{
HAIXIA CHANG,${ }^{1}$ VEHBI E. PAKSOY,${ }^{2}$ and FUZHEN ZHANG ${ }^{2 *}$ \\ Communicated by Q.-W. Wang
}

\begin{abstract}
Considering $n \times n \times n$ stochastic tensors $\left(a_{i j k}\right)$ (i.e., nonnegative hypermatrices in which every sum over one index $i, j$, or $k$, is 1$)$, we study the polytope $\left(\Omega_{n}\right)$ of all these tensors, the convex set $\left(L_{n}\right)$ of all tensors in $\Omega_{n}$ with some positive diagonals, and the polytope $\left(\Delta_{n}\right)$ generated by the permutation tensors. We show that $L_{n}$ is almost the same as $\Omega_{n}$ except for some boundary points. We also present an upper bound for the number of vertices of $\Omega_{n}$.
\end{abstract}

\section{IntRoduction}

A square matrix is doubly stochastic if its entries are all nonnegative and each row and column sum is 1. A celebrated result known as Birkhoff's theorem about doubly stochastic matrices (see, e.g., [8, p. 549]) states that an $n \times n$ matrix is doubly stochastic if and only if it is a convex combination of some $n \times n$ permutation matrices. Considered as elements in $\mathbb{R}^{n^{2}}$, the $n \times n$ doubly stochastic matrices form a polytope $\left(\omega_{n}\right)$. The Birkhoff's theorem says that the polytope $\omega_{n}$ is the same as the polytope $\left(\delta_{n}\right)$ generated by the permutation matrices. A traditional proof of this result is to make use of a lemma which ensures that every doubly stochastic matrix has a positive diagonal (see, e.g., [8, Lemma 8.7.1, p. 548]). By a "diagonal of an $n$-square matrix" we mean a set of $n$ entries taken from different rows and columns. The $n$-square doubly stochastic matrices having a positive diagonal form a polytope $\left(l_{n}\right)$ too. Apparently, $\delta_{n} \subseteq l_{n} \subseteq \omega_{n}$. Birkhoff's theorem asserts that the three polytopes $\omega_{n}, l_{n}$, and $\delta_{n}$ coincide.

Copyright 2016 by the Tusi Mathematical Research Group.

Received Sep. 18, 2015; Accepted Nov. 26, 2015.

${ }^{*}$ Corresponding author.

2010 Mathematics Subject Classification. Primary 15B51; Secondary 52B11.

Keywords. doubly stochastic matrix, extreme point, polytope, stochastic semi-magic cube, stochastic tensor. 